\title{
ON THE ABSOLUTE HARMONIC SUMMABILITY OF FOURIER SERIES
}

\section{O. P. VARSHNEY}

1.1. Let $\sum a_{n}$ be a given infinite series with the sequence of partial sums $\left\{s_{n}\right\}$. Let the sequence $\left\{t_{n}\right\}$ be defined by

$$
\begin{aligned}
t_{n}=\frac{(n+1)^{-1} s_{0}+n^{-1} s_{1}+\cdots+s_{n}}{} & \\
& P_{n} \\
& \left(P_{n}=1+\frac{1}{2}+\cdots+\frac{1}{n+1}\right) .
\end{aligned}
$$

The series $\sum a_{n}$ is defined to be summable by harmonic means if the sequence $\left\{t_{n}\right\}$ tends to a limit as $n \rightarrow \infty$ [2]. If the series $\sum\left|t_{n}-t_{n-1}\right|$ is convergent, we say that the series is absolutely harmonic summable. It is known that this method of summability is absolutely regular and implies absolute Cesàro summability of every positive order [1].

1.2. Let $f(t)$ be a periodic function with period $2 \pi$, and integrable $(L)$ over $(-\pi, \pi)$. Let the Fourier series associated with $f(t)$ be

$$
\frac{1}{2} a_{0}+\sum_{1}^{\infty}\left(a_{n} \cos n t+b_{n} \sin n t\right) \equiv \sum u_{n}
$$

We write

$$
\begin{aligned}
\phi(t) & =f(x+t)+f(x-t)-2 f(x), \\
\alpha(t) & =\sum_{k=0}^{\infty}(k+1)^{-1} \cos k t \\
\beta(t) & =\sum_{k=0}^{\infty}(k+1)^{-1} \sin k t \\
\alpha_{n} & =\int_{0}^{\pi} \phi(t) \alpha(t) \cos n t d t \\
\beta_{n} & =\int_{0}^{\pi} \phi(t) \beta(t) \sin n t d t .
\end{aligned}
$$

Furthermore, $A$ will denote an absolute positive constant and we will write $A+A=A$ and $A-A=A$.

Received by the editors August 27, 1959 and, in revised form, October 5, 1959. 
Zygmund [4] has shown that if $f(x)$ is of bounded variation and satisfies $|f(x+h)-f(x)| \leqq A \log ^{-2-\eta}(1 / h),(\eta>0,0 \leqq x \leqq 2 \pi)$, then the series (1.2.1) converges absolutely. Salem [3] has proved that this theorem is best possible in the sense that $\eta$ cannot be replaced by zero.

The following theorem on the absolute harmonic summability of the series (1.2.1) is analogous to the theorem of Zygmund.

THEOREM. If $f(x)$ is of bounded variation and satisfies

$$
|f(x+h)-f(x)| \leqq A \log ^{-1-\epsilon}\left(\frac{1}{h}\right), \quad(\epsilon>0,0 \leqq x \leqq 2 \pi),
$$

then the series (1.2.1) is absolutely harmonic summable.

2. Proof of the theorem. Using (1.1.1) and (1.2.1) we have

$$
\begin{aligned}
t_{n}-t_{n-1}= & \sum_{k=0}^{n-1}\left(\frac{P_{k}}{P_{n}}-\frac{P_{k-1}}{P_{n-1}}\right) u_{n-k} \\
= & \frac{1}{\pi} \int_{0}^{\pi} \phi(t) \sum_{k=0}^{n-1}\left(\frac{P_{k}}{P_{n}}-\frac{P_{k-1}}{P_{n-1}}\right) \cos (n-k) t d t \\
= & \frac{1}{\pi P_{n} P_{n-1}} \int_{0}^{\pi} \phi(t) \sum_{k=0}^{n-1}\left(\frac{P_{n}}{k+1}-\frac{P_{k}}{n+1}\right) \cos (n-k) t d t \\
= & \frac{1}{\pi P_{n} P_{n-1}} \int_{0}^{\pi} \phi(t)\left\{\sum_{k=0}^{\infty} \frac{P_{n}}{k+1}-\sum_{k=n}^{\infty} \frac{P_{n}}{k+1}-\sum_{k=0}^{n-1} \frac{P_{k}}{n+1}\right\} \\
& \cdot \cos (n-k) t d t .
\end{aligned}
$$

Hence

$$
\begin{aligned}
\pi\left|t_{n}-t_{n-1}\right| & \leqq \\
\qquad & \frac{1}{P_{n-1}}\left|\int_{0}^{\pi} \phi(t)\left(\sum_{k=0}^{\infty} \frac{\cos (n-k) t}{k+1}\right) d t\right| \\
& +\frac{1}{P_{n-1}}\left|\int_{0}^{1 / n} \phi(t)\left(\sum_{k=n}^{\infty} \frac{\cos (n-k) t}{k+1}\right) d t\right| \\
& +\frac{1}{(n+1) P_{n} P_{n-1}}\left|\int_{0}^{1 / n} \phi(t)\left(\sum_{k=0}^{n-1} P_{k} \cos (n-k) t\right) d t\right| \\
& +\frac{1}{P_{n-1}}\left|\int_{1 / n}^{\pi} \phi(t)\left\{\sum_{k=n}^{\infty} \frac{\cos (n-k) t}{k+1}+\sum_{k=0}^{n-1} \frac{P_{k} \cos (n-k) t}{(n+1) P_{n}}\right\} d t\right| \\
= & g_{1}(n)+g_{2}(n)+g_{3}(n)+g_{4}(n), \text { say. }
\end{aligned}
$$


Therefore

(2.2) $\pi \sum\left|t_{n}-t_{n-1}\right| \leqq \sum g_{1}(n)+\sum g_{2}(n)+\sum g_{3}(n)+\sum g_{4}(n)$.

Now

$$
\begin{aligned}
\sum \mathfrak{g}_{1}(n)= & \sum \frac{1}{P_{n-1}}\left|\int_{0}^{\pi} \phi(t) \sum_{k=0}^{\infty} \frac{\cos (n-k) t}{k+1} d t\right| \\
= & \sum \frac{1}{P_{n-1}}\left|\int_{0}^{\pi} \phi(t)\left\{\sum_{k=0}^{\infty} \frac{\cos n t \cos k t}{k+1}+\sum_{k=0}^{\infty} \frac{\sin n t \sin k t}{k+1}\right\} d t\right| \\
& \leqq \sum \frac{1}{P_{n-1}}\left|\int_{0}^{\pi} \phi(t) \alpha(t) \cos n t d t\right| \\
& +\sum \frac{1}{P_{n-1}}\left|\int_{0}^{\pi} \phi(t) \beta(t) \sin n t d t\right| \\
= & \sum \frac{\left|\alpha_{n}\right|+\left|\beta_{n}\right|}{P_{n-1}} .
\end{aligned}
$$

Since $1 /(n+1)$ decreases steadily to zero, $\sum_{k=n}^{\infty}(\cos (n-k) t) /(k+1)$ converges for $t \neq 0$; and its sum does not exceed $4 /(n+1)\left|1-e^{-i t}\right|$ in absolute value, we have

$$
\begin{aligned}
\sum g_{2}(n) & =\sum \frac{1}{P_{n-1}}\left|\int_{0}^{1 / n} \phi(t) \sum_{k=n}^{\infty} \frac{\cos (n-k) t}{k+1} d t\right| \\
& \leqq A \sum \frac{1}{P_{n-1}}\left|\int_{0}^{1 / n} \frac{1}{(n+1) t \log ^{1+\epsilon}(1 / t)} d t\right| \\
& \leqq A \sum \frac{1}{(n+1) \log ^{1+\epsilon} n} \leqq A,
\end{aligned}
$$

in view of the fact that (1.2.2) implies

Again, by (2.5)

$$
|\phi(t)| \leqq A \log ^{-1-\epsilon}\left(\frac{1}{t}\right), \quad(0 \leqq t \leqq \pi) .
$$

$$
\begin{aligned}
\sum g_{3}(n) & =\sum \frac{1}{(n+1) P_{n} P_{n-1}}\left|\int_{0}^{1 / n} \phi(t)\left(\sum_{k=0}^{n-1} P_{k} \cos (n-k) t\right) d t\right| \\
& \leqq A \sum \frac{1}{\log n} \int_{0}^{1 / n} \frac{1}{\log ^{1+\epsilon}(1 / t)} d t \\
& \leqq A \sum \frac{1}{n \log ^{2+\epsilon} n} \leqq A .
\end{aligned}
$$


1960] ON THE ABSOLUTE HARMONIC SUMMABILITY OF FOURIER SERIES 591

Since $1 / n(n+1)$ decreases steadily to zero,

$$
\sum_{n}^{\infty}\left(\sin \left(n-k+\frac{1}{2}\right) t\right) / k(k+1)
$$

converges, and its sum does not exceed $4 / n(n+1)\left|1-e^{-i t}\right|$ in absolute value, we have by Abel's transformation

$$
\begin{aligned}
\sum_{k=n}^{\infty} \frac{\cos (n-k) t}{k+1} & =\frac{1}{(n+1)}+\frac{1}{(n+2)} \cos t+\frac{1}{(n+3)} \cos 2 t+\cdots \\
& =\frac{1}{2(n+1)}+\left(\frac{1}{(n+1)}-\frac{1}{(n+2)}\right) \frac{1}{2} \\
& +\left(\frac{1}{(n+2)}-\frac{1}{(n+3)}\right)\left(\frac{1}{2}+\cos t\right)+\cdots \\
& =\frac{1}{2(n+1)}+\sum_{k=n}^{\infty} \frac{1}{k(k+1)} \frac{\sin (n-k+1 / 2) t}{2 \sin (t / 2)} \\
& =\frac{1}{2(n+1)}+O\left(\frac{1}{n^{2} t^{2}}\right), \quad \text { for } t \geqq \frac{1}{n} .
\end{aligned}
$$

Since $\sum_{0}^{n-1} \sin (n-k+1 / 2) t /(k+1)=O(1+\log (1 / t))$ for $t=1 / n$, we have by Abel's transformation,

$$
\begin{aligned}
\sum_{k=0}^{n-1} P_{k} \cos (n- & k) t \\
= & P_{0} \cos n t+P_{1} \cos (n-1) t+\cdots+P_{n-1} \cos t \\
= & \left(P_{n-1}-P_{n-2}\right)\left(\frac{1}{2}+\cos t\right)+\cdots \\
& +\left(P_{1}-P_{0}\right)\left(\frac{1}{2}+\cos t+\cdots+\cos (n-1) t\right) \\
& +P_{0}\left(\frac{1}{2}+\cos t+\cdots+\cos n t\right)-\frac{1}{2} P_{n-1} \\
= & \sum_{k=0}^{n-1} \frac{1}{k+1} \frac{\sin (n-k+1 / 2) t}{2 \sin (t / 2)}-\frac{1}{2} P_{n-1} \\
= & O\left(\frac{1+\log (1 / t)}{t}\right)-\frac{1}{2} P_{n-1} .
\end{aligned}
$$

Now, from (2.5), (2.7) and (2.8) we obtain 


$$
\begin{aligned}
& \sum g_{4}(n)=\sum \frac{1}{P_{n-1}} \mid \int_{1 / n}^{\pi} \phi(t)\left\{\sum_{k=n}^{\infty} \frac{\cos (n-k) t}{k+1}\right. \\
& \left.+\sum_{k=0}^{n-1} \frac{P_{k} \cos (n-k) t}{(n+1) P_{n}}\right\} d t \mid \\
& \leqq \sum \frac{A}{P_{n-1}} \int_{1 / n}^{\pi}|\phi(t)| \frac{1}{n^{2} t^{2}} d t \\
& +\sum \frac{A}{(n+1) \log ^{2} n} \int_{1 / n}^{\pi}|\phi(t)| \frac{1+\log (1 / t)}{t} d t \\
& +\sum \frac{A}{\log n} \int_{1 / n}^{\pi}|\phi(t)|\left(\frac{1}{(n+1)}-\frac{P_{n-1}}{(n+1) P_{n}}\right) d t \\
& \leqq A \sum \frac{1}{n^{2} \log n} \int_{1 / n}^{\pi} \frac{d t}{t^{2} \log ^{1+\epsilon}(1 / t)} \\
& +A \sum \frac{1}{n \log ^{2} n} \int_{1 / n}^{\pi} \frac{d t}{t \log ^{e}(1 / t)} \\
& +A \sum \frac{1}{n^{2} \log ^{2} n} \int_{1 / n}^{\pi} \frac{d t}{\log ^{1+\epsilon}(1 / t)} \\
& \leqq A \sum \frac{1}{n(\log n)^{1+\epsilon}} \\
& \leqq A \text {. }
\end{aligned}
$$

Combining (2.2), (2.3), (2.4), (2.6) and (2.9) we find that, in order to prove our theorem, we need only show that

$$
\sum_{n} \frac{\left|\alpha_{n}\right|+\left|\beta_{n}\right|}{P_{n-1}}<\infty \text {. }
$$

Now $\alpha(t)$ and $\beta(t)$ are continuous for $\eta \leqq t \leqq \pi$ and their absolute values when $0<t \leqq \eta$ are each less than $A \log (1 / t)$. The constants $\alpha_{n}$ and $\beta_{n}$ are thus the Fourier coefficients of an even and odd function respectively and each of these functions belongs to $L^{2}$. The Fourier series of $\phi(t+h) \alpha(t+h)-\phi(t-h) \alpha(t-h)$ is

$$
-\frac{4}{\pi} \sum_{n=1}^{\infty} \alpha_{n} \sin n t \sin n h .
$$

It follows from Parseval's theorem that 
1960] ON THE ABSOLUTE HARMONIC SUMMABILITY OF FOURIER SERIES 593

$$
\begin{aligned}
\sum_{n=1}^{\infty} \alpha_{n}^{2} \sin ^{2} n h \leqq & A \int_{0}^{\pi}\{\phi(t+h) \alpha(t+h)-\phi(t-h) \alpha(t-h)\}^{2} d t \\
\leqq & A \int_{0}^{\pi} \alpha^{2}(t+h)|\phi(t+h)-\phi(t-h)|^{2} d t \\
& +A \int_{0}^{\pi} \phi^{2}(t-h)|\alpha(t+h)-\alpha(t-h)|^{2} d t \\
= & J_{1}(h)+J_{2}(h) .
\end{aligned}
$$

We first consider $J_{2}(h)$.

$J_{2}(h)=A \int_{0}^{\pi} \phi^{2}(t-h)|\alpha(t+h)-\alpha(t-h)|^{2} d t$

$$
\begin{aligned}
\leqq A\left\{\int_{-h}^{h} \phi^{2}(t) \alpha^{2}(t+2 h) d t\right. & +\int_{-h}^{h} \phi^{2}(t) \alpha^{2}(t) d t \\
& \left.\quad+\int_{h}^{\pi} \phi^{2}(t)|\alpha(t+2 h)-\alpha(t)|^{2} d t\right\} \\
= & J_{2,1}(h)+J_{2,2}(h)+J_{2,3}(h) .
\end{aligned}
$$

Using (2.5) we get

$$
\begin{aligned}
J_{2,1}(h) & \leqq A \frac{1}{\log ^{2+2 \epsilon}(1 / h)} \int_{-h}^{h} \log ^{2}\left(\frac{1}{t+2 h}\right) d t \\
& \leqq \frac{A}{\log ^{2 \epsilon}(1 / h)} \int_{-h}^{h} d t \leqq \frac{A h}{\log ^{2 \epsilon}(1 / h)}
\end{aligned}
$$

and similarly

$$
\begin{aligned}
J_{2,2}(h) & \leqq A \int_{0}^{h} \phi^{2}(t) \alpha^{2}(t) d t \\
& \leqq A \int_{0}^{h} \log ^{-2-2 \epsilon}\left(\frac{1}{t}\right) \cdot \log ^{2}\left(\frac{1}{t}\right) d t \\
& \leqq A h / \log ^{2 \epsilon}(1 / h) .
\end{aligned}
$$

Finally,

$$
\begin{aligned}
J_{2,3}(h) & \leqq A h^{2} \int_{h}^{\pi} \phi^{2}(t)\left\{\alpha^{\prime}(t+2 \theta h)\right\}^{2} d t \quad(0<\theta<1) \\
& \leqq A h^{2} \int_{h}^{\pi} \log ^{-2-2 \epsilon}\left(\frac{1}{t}\right) \frac{d t}{(t+2 \theta h)^{2}} \\
& \leqq A h / \log ^{2 e}(1 / h) .
\end{aligned}
$$


Now setting $h=(\pi / 2 N)$ and collecting (2.11), (2.12), (2.13) and (2.14) we find that

$$
J_{2}(h) \leqq \frac{A}{N \log ^{2 e} N} \cdot
$$

Since $f$ is continuous and of bounded variation, it is clear that $\phi$ is also continuous and of bounded variation. Let $\omega(\delta)$ be the modulus of continuity of $\phi$, and $V$ the total variation of $\phi$ over $(0,2 \pi)$. We start from the inequality

$$
\begin{aligned}
\sum_{k=1}^{2 N}\left\{\alpha\left(t+\frac{k \pi}{N}\right)\right\}^{2}\left[\phi\left(t+\frac{k \pi}{N}\right)-\phi\left(t+(k-1) \frac{\pi}{N}\right)\right]^{2} \\
\leqq A \log ^{2} \frac{1}{t} \sum_{k=1}^{2 N}\left[\phi\left(t+k \frac{\pi}{N}\right)-\phi\left(t+(k-1) \frac{\pi}{N}\right)\right]^{2} \\
\leqq A \log ^{2} \frac{1}{t} \omega\left(\frac{\pi}{N}\right) \sum_{k=1}^{2 N}\left|\phi\left(t+\frac{k \pi}{N}\right)-\phi\left(t+(k-1) \frac{\pi}{N}\right)\right| \\
\leqq A \log ^{2} \frac{1}{t} \frac{V}{\log ^{1+\epsilon} N},
\end{aligned}
$$

which we integrate over $(0, \pi)$. On account of the periodicity, replacing $x$ by $x+\xi$ does not affect the value of the integral, and so all integrals formed from the left hand side are equal. Hence we have,

$$
\begin{aligned}
2 N \int_{0}^{\pi}\left\{\alpha\left(t+\frac{\pi}{2 N}\right)\right\}^{2}[\phi(t & \left.\left.+\frac{\pi}{2 N}\right)-\phi\left(t-\frac{\pi}{2 N}\right)\right]^{2} d t \\
& \leqq \frac{A}{\log ^{1+\epsilon} N} \int_{0}^{\pi} \log ^{2} \frac{1}{t} d t \\
& \leqq \frac{A}{\log ^{1+\epsilon} N} .
\end{aligned}
$$

Hence

$$
J_{1}\left(\frac{\pi}{2 N}\right) \leqq \frac{A}{N \log ^{1+\epsilon} N} .
$$

Combining (2.15) and (2.16) we find that

$$
\sum_{n=1}^{\infty} \alpha_{n}^{2} \sin ^{2}\left(\frac{n \pi}{2 N}\right) \leqq \frac{A}{N \log ^{2 \epsilon} N} \quad(\epsilon<1) .
$$

Taking $N=2^{p}$, we get 
I960] ON THE ABSOLUTE HARMONIC SUMMABILITY OF FOURIER SERIES 595

$$
\begin{aligned}
\sum_{n=2^{\nu-1}+1}^{2 \nu} \alpha_{n}^{2} & \leqq 2 \sum_{n=2^{\nu-1}+1}^{2 \nu} \alpha_{n}^{2} \sin ^{2}\left(\frac{n \pi}{2^{\nu+1}}\right) \\
& \leqq 2 \sum_{n=1}^{\infty} \alpha_{n}^{2} \sin ^{2}\left(\frac{n \pi}{2^{\nu+1}}\right) \\
& \leqq A\left(2^{-\nu} \cdot \nu^{-2 \epsilon}\right) .
\end{aligned}
$$

Therefore, applying Schwarz's inequality we get

$$
\begin{aligned}
\sum_{n=2^{\nu-1}+1}^{2^{\nu}}\left|\alpha_{n}\right| \log ^{-1} n & \leqq\left\{\sum_{n=2^{\nu-1}+1}^{2^{\nu}} \alpha_{n}^{2}\right\}^{1 / 2}\left\{\sum_{n=2^{\nu-1}+1}^{2^{\nu}} \log ^{-2} n\right\}^{1 / 2} \\
& \leqq A\left\{2^{-\nu / 2} \cdot \nu^{-\epsilon}\right\} 2^{(\nu-1) / 2} \cdot(\nu-1)^{-1} \\
& \leqq A / \nu^{1+\epsilon} .
\end{aligned}
$$

A similar relation holds in the case of $\beta_{n}$. Hence

$$
\begin{aligned}
\sum_{n=1}^{\infty} \frac{\left|\alpha_{n}\right|+\left|\beta_{n}\right|}{P_{n-1}} & \leqq A \sum_{n=1}^{\infty} \frac{\left|\alpha_{n}\right|}{\log n}+A \sum_{n=1}^{\infty} \frac{\left|\beta_{n}\right|}{\log n} \\
& =A \sum_{\nu=1}^{\infty} \sum_{n=2^{\nu-1}+1}^{2^{\nu}} \frac{\left|\alpha_{n}\right|}{\log n}+A \sum_{\nu=1}^{\infty} \sum_{n=2^{\nu-1}+1}^{2^{\nu}} \frac{\left|\beta_{n}\right|}{\log n} \\
& \leqq A \sum_{\nu=1}^{\infty} \frac{1}{\nu^{1+\epsilon}} \leqq A ;
\end{aligned}
$$

and thus the proof of the theorem is complete.

I am much indebted to Professor M. L. Misra for his kind interest and advice in the preparation of this paper. I am also thankful to the referee for drawing my attention to the paper of Salem.

\section{REFERENCES}

1. L. McFadden, Absolute Nörlund summability, Duke Math. J. vol. 9 (1942) pp. 168-207.

2. M. Riesz, Sur l'équivalence de certaines methodes de sommation, Proc. London Math. Soc. vol. 22 (1924) pp. 412-419.

3. R. Salem, On a theorem of Zygmund, Duke Math. J. vol. 10 (1943) pp. 23-31.

4. A. Zygmund, Sur la convergence absolue des séries de Fourier, J. London Math. Soc. vol. 3 (1928) pp. 194-196.

UNIVERSITY OF SAUgar, SAgar, India 